\title{
Myopia Control with Combination Low-Dose Atropine and Peripheral Defocus Soft Contact Lenses: A Case Series
}

\author{
Nir Erdinesta, ${ }^{a} \quad$ Naomi London ${ }^{c} \quad$ Nadav Levinger ${ }^{a, d} \quad$ Yair Morad $^{b, e}$ \\ aDepartment of Ophthalmology, Hadassah-Hebrew University Medical Center, Jerusalem,

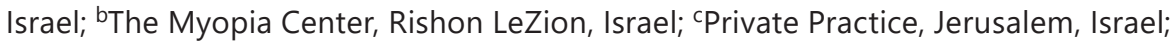 \\ ${ }^{\mathrm{d} D e p a r t m e n t}$ of Opthalmology, Enaim Refractive Surgery Center, Jerusalem, Israel; \\ eDepartment of Ophthalmology, Assaf Harofeh Medical Center, Zerifin, Israel
}

\section{Keywords}

Myopia progression · Myopia control · Atropine · Peripheral defocus · Soft contact lenses

\begin{abstract}
The goal of this retrospective case series is to demonstrate the effectivity of combination lowdose atropine therapy with peripheral defocus, double concentric circle design with a center distance soft contact lenses at controlling myopia progression over 1 year of treatment. Included in this series are 3 female children aged 8-10 years with progressing myopia averaging $-4.37 \pm 0.88 \mathrm{D}$ at the beginning of treatment. Their average annual myopic progression during the 3 years prior to therapy was $1.12 \pm 0.75 \mathrm{D}$. They had not attempted any myopia control treatments prior to this therapy. The children were treated with a combination of $0.01 \%$ atropine therapy with spherical peripheral defocus daily replacement soft lenses MiSight ${ }^{\circledR} 1$ day (Cooper Vision, Phoenix, AZ, USA). They underwent cycloplegic refraction, and a slit-lamp evaluation every 6 months which confirmed no adverse reactions or staining was present. Each of the 3 children exhibited an average of $0.25 \pm 0.25 \mathrm{D}$ of myopia progression at the end of 1 year of treatment. To the best of the authors' knowledge, this is the first published study exhibiting that combining low-dose atropine and peripheral defocus soft contact lenses is effective at controlling children's moderate to severe myopia progression during 1 year of therapy.
\end{abstract}

\section{Karger $\stackrel{2}{*}$}




\section{Introduction}

Myopia is increasing worldwide both in incidence as well as magnitude [1]. Both genetic and environmental factors have an influence on myopia occurrence and progression [2], and some of these factors seem to be interconnected $[3,4]$. The potential myopia-induced pathological sequelae such as retinal detachment, macular degeneration, glaucoma, and cataract have encouraged extensive research in an attempt to find treatments that will halt progression [2-4].

Of the various therapies found effective, to date the leading treatments are atropine therapy, peripheral myopic defocus therapies including orthokeratology and soft bifocal or multifocal center distance contact lenses, increased sunlight, and bifocal or progressive addition spectacle lenses [1]. While each treatment option is considered effective in certain cases, the additive effect of combination therapies has just begun to be extensively researched.

This case series demonstrates the effectivity of combining atropine and peripheral defocus soft contact lens therapy on 3 children over the course of 1 year. To the best of our knowledge, this is the first report on 1 year of combination low-dose atropine $(0.01 \%)$ and peripheral defocus soft contact lenses therapy in children with moderately progressing myopia.

\section{Methods}

Presented here is a retrospective case series of 3 female children between the ages of 8-10 years with progressing myopia. It should be noted that the subjects are not siblings. At the initial exam, the children had an average cycloplegic spherical equivalent refraction (SER) of $-4.37 \pm 0.88 \mathrm{D}$ with $<1$ diopter of astigmatism. The average annual myopia progression during the 3 years prior to this consultation was $1.12 \pm 0.13 \mathrm{D}$ three years earlier, $0.83 \pm 0.16$ and $0.79 \pm 0.2 \mathrm{D}, 2$ years and 1 year earlier, respectively. Each of their parents suffers from myopia above $4.00 \mathrm{D}$, and understanding the genetic component they probably had bequeathed to their offspring, they were eager to try and control the progression in their children.

Options for decreasing the progression of myopia were discussed with the parents and the children, including the use of low-dose atropine $(0.01 \%)$ with or without progressive addition spectacle lenses, orthokeratology, or soft bifocal contact lenses. The risks and benefits associated with each option were discussed. It was made clear that each option had researched published data available that it may slow myopic progression. They chose combination $0.01 \%$ atropine with periphery defocus soft contact lenses. These children had not attempted any myopia control treatments prior to this discussion nor did any have previous contact lens experience.

The binocular status of the 3 children indicated no amblyopia, strabismus, or accommodative dysfunctions. Near point of convergence was within $5 \mathrm{~cm}$ of the nose for all 3. Two children exhibited a near point esophoria (3 esophoria and 5 esophoria) and the third exhibited orthophoria. The children had no systemic conditions that would affect ocular health or lens wear such as diabetes or autoimmune conditions. They all exhibited ocular health with no contraindications to contact lens wear. The children did not have dry eyes or tarsal papillae.

The horizontal visual iris diameter was between 11.7 and $11.9 \mathrm{~mm}$ in all 3 children. Their mean corneal keratometry was $7.70 \pm 0.1 \mathrm{~mm}$. The lenses prescribed were spherical daily replacement soft lenses made of omafilcon A (MiSight ${ }^{\circledR} 1$ day, Cooper Vision, Phoenix, AZ, USA) with a $14.2 \mathrm{~mm}$ diameter and base curve $8.7 \mathrm{~mm}$. The lens design includes 2 sets of alternating concentric rings of center distance and $\mathrm{a}+2.00 \mathrm{D}$ addition surrounding ring. They

\section{Karger'}


were prescribed within their suggested indicated use for the correction of myopia to be worn at least $10 \mathrm{~h}$ per day 6 days a week. The prescription given was calculated as the full spherical equivalent refractions, and then the vertex distance was calculated as required.

The lenses were well centered on the corneas and movement upon blink was approximately 1-2 mm with minimal movement during peripheral gaze. The parents and children were instructed how to properly handle the contact lenses including how to insert and remove them. The children reported the lenses were comfortable, and their visual acuity (VA) was not significantly inferior compared with their spectacles.

Concurrently, the children were prescribed $0.01 \%$ atropine. It was prepared by a pharmacy chain (Super-Pharm Professional, Petah Tikva, Israel). The eye drops were packaged in opaque (to protect from photodegrading), $10.0 \mathrm{~mL}$ sterile bottles with $5 \mathrm{~mL}$ volume with benzalkonium chloride $0.01 \%$ as the preservative. The atropine sulfate bottles were stored no longer than 21 days in $4^{\circ} \mathrm{C}$. The parents were instructed to instill 1 drop of atropine in each eye daily before bedtime.

Follow-up visits to confirm ocular health and that there were no adverse reactions were conducted at 1 week, 1 month, and 3 months after dispensing. The children were followed every 6 months when a slit-lamp evaluation and cycloplegic refraction were conducted. Refraction was measured after installation of 2 drops of tropicamide 1\%, 1 drop instilled at 5 min intervals.

All SER measurements were performed post mydriasis by the same practitioner in the same examination room using identical ambient lighting. Distance VA was measured monocularly using a Snellen chart.

\section{Ethical Principles}

The parents provided written consent to disclose the details and results of the children's treatment.

\section{Results}

The SER at the beginning of treatment of each of the 3 children was $-4.37 \pm 0.88 \mathrm{D}$ $(-4.25$ and $-4.00 \mathrm{D},-3.50$ and $-3.75 \mathrm{D}$, and -5.25 and $-5.00 \mathrm{D}$ in the right and left eye of each child, respectively). Their VA was $6 / 6,6 / 5$, and 6/6, respectively. The average change in the SER measured at the end of the year of treatment was $0.25 \pm 0.25 \mathrm{D}$ (plano and -0.25 $\mathrm{D},-0.50$ and $-0.25 \mathrm{D}$, and -0.25 and plano in the right and left eye of each child, respectively, Table 1).

The VA and binocular status did not change during the year nor were there any adverse corneal reactions noted including staining, infiltrates, or erosions. There was no tarsal or bulbar conjunctival erythema and no noted giant papillary conjunctivitis.

There was no change in refraction noted at the 6-month visit. The children are continuing to wear the lenses at the time of submission, 6 months after the end of this study.

\section{Discussion}

This case series is the first to demonstrate the effectivity of combining $0.01 \%$ atropine and peripheral defocus soft contact lens treatment at halting myopia progression. The average annual progression of these children during the 3 years prior to therapy was $1.12 \pm 0.75 \mathrm{D}$ whereas the average increase at the end of the year of therapy was $0.25 \pm 0.25 \mathrm{D}$, exhibiting a pronounced effectivity.

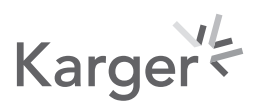




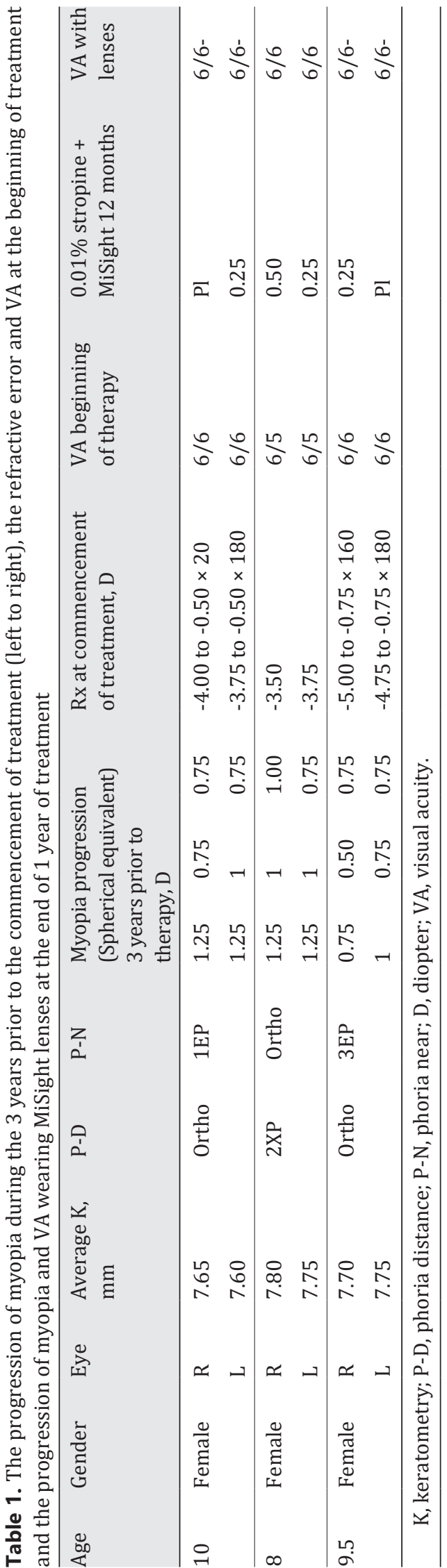

\section{Karger'}


There is an added possible bias that needs to be acknowledged, namely these 3 children were relatively active and played outdoors a lot. They may have had the additional advantage of the sunlight which is a myopia inhibitor [1].

Research has monitored myopia progression of children wearing MiSight lenses as a monotherapy treatment over the course of 1-3 years. The largest study in the published literature to date followed 109 children in which the average increase in myopia after year 1 was $0.27 \pm 0.07 \mathrm{D}$. In this same study the effectivity did not improve over the second and third year of treatment but was still more effective than the control group undergoing no treatment [5].

Atropine therapy has been shown to be effective in many studies, specifically $0.01 \%$ had been acknowledged in the literature at the commencement of this study to be the preferred concentration and most potent monotherapy to decrease myopia progression [2, 6-10]. Additionally, it has been shown to have an advantage of exhibiting relatively low rebound effect after cessation of therapy [11]. The decrease in progression during the first year of $0.01 \%$ atropine monotherapy has been shown to be variable. In the Low-Concentration Atropine for Myopia Progression (LAMP) study the progression at year 1 was 0.59 $\pm 0.61 \mathrm{D}$ [2], in the Larkin et al. [6] $0.01 \%$ atropine multiethnic study $0.20 \pm 0.8 \mathrm{D}$ and the Atropine for the Treatment of Myopia 2 (ATOM2) found 0.43 D [12] although the heterogeneity of these studies makes it difficult to make an accurate comparison. Some of these studies extended into second and third years of therapy and have exhibited improved effectivity in the following years, suggesting these children may benefit as well from longer therapy $[2,12]$. The variability of first-year atropine therapy encouraged a combination approach in these particular children where there was a desire to halt progression as quickly as possible, as myopia was increasing at quite a moderate rate.

The Bifocal Lenses in Nearsighted Kids (BLINK) study, which began in 2017, compared the effectivity of identical design single-vision, a center distance multifocal soft contact lenses with $\mathrm{a}+1.50 \mathrm{D}$ addition and a center distance multifocal with $\mathrm{a}+2.50 \mathrm{D}$ addition [13]. The data so far suggest that for the peripheral blur to be effective, there may be either a dioptric threshold or a minimal area of visual field required for the inhibitory effect $[3,4]$. This supports applying a lens such as the MiSight used in this study which incorporates 2 concentric circles of peripheral blur thus assuring a larger retinal area of peripheral defocus as well as in both photopic and scotopic circumstances when pupil size changes.

Myopia progression is associated with axial elongation and even considered a principal ocular component for endeavoring to decrease progression to prevent the potential physiological consequences. The authors acknowledge the probable axial elongation with increase in myopia, even if not linearly [12]. Research has shown it generally occurs without significant change in other parameters or physiology. Not including measurements in this study does not indicate their lack of significance [12].

The multifactorial nature of myopia imposes a challenge to effective treatment. Therapies to date do not intervene with the genetic component but are more targeted to the biological or physiological elements. Though the precise mechanisms of action of atropine therapy remain elusive, the proposed options are five-fold. First, atropine is a reversible competitive antagonist, with an affinity for all subtypes of the muscarinic receptors in the sclera which cause proliferation of scleral fibroblasts and axial elongation [8]. It has been shown to reduce epidermal growth factor receptor activity in scleral fibroblasts and to inhibit choroidal thinning caused by hyperopic retinal defocus [8]. Atropine increases the release of the neurotransmitter dopamine which hinders ocular eye growth [8]. Last, atropine has been observed to intervene in other biological mechanisms including the retinal signaling pathways responding to environmental cues

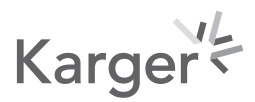


and in the retinal pigment epithelium when relaying ocular growth regulatory signals from the retina to sclera [8].

The mechanism underlying the effectivity of optical peripheral myopic defocus treatment is yet undetermined. Hypotheses include reducing an accommodative lag [14] and possibly halting the excessive expansion of Bruch's membrane [15]. It is as yet undetermined the exact area of the retina, the surface area required, or the depth of myopic defocus required for maximum efficacy. The consideration in this study was to combine treatments to control myopia progression from 2 different approaches. Both the compound optical (peripheral defocus) and biological (atropine) treatments complement each other and potentially may prove more effective than when administered in isolation.

Further, studies over a longer period of time are needed to clarify whether this modality is effective to the same degree over a few years and whether a different schedule of contact lenses wear would affect the outcome. Additional research will help discover whether an adjustment to the atropine concentration is required in different cases of myopia progression and whether the rebound effect when discontinuing the atropine treatment differs to that with monotherapy atropine.

\section{Conclusions}

Combination low-dose atropine and peripheral defocus daily replacement soft contact lenses are effective at controlling myopia during 1 year of therapy in children exhibiting moderate progressive increase.

\section{Acknowledgements}

Dr. Nir Erdinest is grateful to the Azrieli Foundation for the award of Pos-doc Azrieli Fellowship and Hadassah-Hebrew University Medical Center for the award of post-doctoral fellows.

\section{Statement of Ethics}

The authors state that this case report was conducted according to good clinical practice and has no ethical conflicts to disclose. Written informed consent was obtained from the legal guardians of the patients for publication of this case and any accompanying images. This report does not contain any personal information that could lead to the identification of the patients.

\section{Conflict of Interest Statement}

The authors of this manuscript do not have any conflicts of interest to declare.

\section{Funding Sources}

This manuscript did not receive any funding.

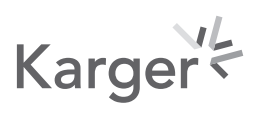




\section{Case Reports in Ophthalmology}

\section{Author Contributions}

Nir Erdinest: patient examination, contact lens fitting and follow-up, data collection, involved in the design, interpretation the data, and manuscript preparation. Naomi London: patient examination, data collection and patient follow-up, and manuscript preparation. Nadav Levinger: manuscript preparation and data collection, Yair Morad: patient examination, follow-up, data collection, involved in the design, revision, and conduct of the study. All the authors approve the final version of the manuscript for publication.

\section{References}

1 Mak CY, Yam JC, Chen LJ, Lee SM, Young AL. Epidemiology of myopia and prevention of myopia progression in children in East Asia: a review. Hong Kong Med J. 2018;24(6):602-9.

2 Yam JC, Jiang Y, Tang SM, Law AKP, Chan JJ, Wong E, et al. Low-concentration atropine for myopia progression (LAMP) study: a randomized, double-blinded, placebo-controlled trial of $0.05 \%, 0.025 \%$, and $0.01 \%$ atropine eye drops in myopia control. Ophthalmology. 2019;126(1):113-24.

3 Tang WC, Leung M, Wong AC, To C-H, Lam CS. Optical interventions for myopia control. Updates on myopia. Singapore: Springer; 2020. p. 289-305.

4 Walline JJ, Lindsley KB, Vedula SS, Cotter SA, Mutti DO, Ng SM, et al. Interventions to slow progression of myopia in children. Cochrane Database Syst Rev. 2020;1(1):CD004916.

5 Chamberlain P, Peixoto-de-Matos SC, Logan NS, Ngo C, Jones D, Young G. A 3-year randomized clinical trial of MiSight lenses for myopia control. Optom Vis Sci. 2019;96(8):556-67.

6 Larkin GL, Tahir A, Epley KD, Beauchamp CL, Tong JT, Clark RA. Atropine 0.01\% eye drops for myopia control in American children: a multiethnic sample across three US sites. Ophthalmol Ther. 2019;8(4):589-98.

7 Kinoshita N, Konno Y, Hamada N, Kanda Y, Shimmura-Tomita M, Kaburaki T, et al. Efficacy of combined orthokeratology and $0.01 \%$ atropine solution for slowing axial elongation in children with myopia: a 2-year randomised trial. Sci Rep. 2020;10(1):1-11.

8 Upadhyay A, Beuerman RW. Biological mechanisms of atropine control of myopia. Eye Contact Lens. 2020; 46(3):129.

9 Wei S, Li S-M, An W, Du J, Liang X, Sun Y, et al. Safety and efficacy of low-dose atropine eyedrops for the treatment of myopia progression in Chinese children: a randomized clinical trial. JAMA Ophthalmol. 2020; 138(11):1178-84.

10 Hieda O, Hiraoka T, Fujikado T, Ishiko S, Hasebe S, Torii H, et al. Efficacy and safety of 0.01\% atropine for prevention of childhood myopia in a 2-year randomized placebo-controlled study. Jpn J Ophthalmol. 2021; $1-11$.

11 Chia A, Chua WH, Wen L, Fong A, Goon YY, Tan D. Atropine for the treatment of childhood myopia: changes after stopping atropine $0.01 \%, 0.1 \%$ and 0.5\%. Am J Ophthalmol. 2014;157(2):451-e1.

12 Chia A, Lu QS, Tan D. Five-year clinical trial on atropine for the treatment of myopia 2: myopia control with atropine 0.01\% eyedrops. Ophthalmology. 2016;123(2):391-9.

13 Walline JJ, Gaume Giannoni A, Sinnott LT, Chandler MA, Huang J, Mutti DO, et al. A randomized trial of soft multifocal contact lenses for myopia control: baseline data and methods. Optom Vis Sci. 2017;94(9):856.

14 Sankaridurg P. Contact lenses to slow progression of myopia. Clin Exp Optom. 2017;100(5):432-7.

15 Wu P-C, Chuang M-N, Choi J, Chen H, Wu G, Ohno-Matsui K, et al. Update in myopia and treatment strategy of atropine use in myopia control. Eye. 2019;33(1):3-13.

\section{Karger'}

